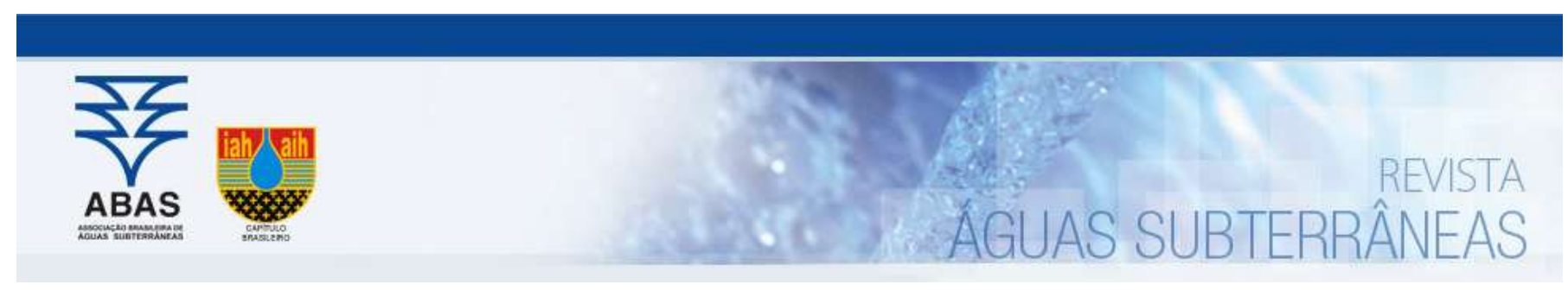

Artigos

\title{
Análise de Desempenho de Modelos de Infiltração Unidimensional de Água no Solo
}

\section{Analysis of Performance of One-dimensional Soil Water Infiltration Models}

\author{
Diogo Botelho Correa de Oliveira ${ }^{1}$; Willames de Albuquerque Soares ${ }^{1}$; Marco Aurelio Calixto Ribeiro de Holanda ${ }^{1}$ \\ 1 Universidade de Pernambuco (UPE), Recife, PE \\ $\triangle$ diogobotelhocorrea@gmail.com,was@poli.br, holandamacr@yahoo.com.br
}

Resumo

Palavras-chave:

Modelos de Infiltração.

Águas Subterrâneas.

Recarga dos lençóis freáticos.

\begin{abstract}
A crescente urbanização e impermeabilização do solo agrava diversos efeitos negativos no ciclo hidrológico da água, impactando diretamente na recarga dos lençóis freáticos e das águas subterrâneas. Em uma região central e relevante da cidade do Recife, objetivou-se analisar o desempenho de seis modelos numéricos de infiltração unidimensional de água no solo: Kostiakov, Horton, Philip, Kostiakov-Lewis, SCS e Swartzendruber, comparando suas modelagens com testes de infiltração de campo, realizados com a utilização de infiltrômetro. Para análise de desempenho utilizaram-se os seguintes parâmetros estatísticos: coeficiente de determinação, razão de desvios, eficiência de modelagem e o erro quadrático médio. Todos se mostraram bastante eficazes em realizar o proposto, destacando-se o de Kostiakov-Lewis, que apresentou excelente desempenho em todos os indicadores estatísticos.
\end{abstract}

Abstract

Keywords:

Infiltration models.

Groundwater.

Recharge of groundwater.

The increasing urbanization and impermeabilization of the soil aggravates several negative effects in the hydrological cycle of the water, directly impacting the recharge of the groundwater and groundwater. In a central and relevant region of the city of Recife, the objective of this study was to analyze the performance of six numerical models of unidimensional water infiltration in the soil: Kostiakov, Horton, Philip, Kostiakov-Lewis, SCS and Swartzendruber, comparing their models with infiltration tests performed using the infiltrator. For performance analysis, the following statistical parameters were used: coefficient of determination, ratio of deviations, modeling efficiency and mean square error. All of them proved to be quite effective in carrying out the proposal, especially Kostiakov-Lewis, who presented excellent performance in all the statistical indicators

DOI: http://dx.doi.org/10.14295/ras.v32i1.28947

\section{INTRODUÇÃO}

A água é um recurso natural de grande relevância nas atividades antrópicas em diversos âmbitos. Seu ciclo possui componentes indispensáveis para a manutenção do equilíbrio passivo entre a urbanização e a natureza. Os processos que compõem o ciclo hidrológico, desde a infiltração, a evapotranspiração até escoamento superficial são dependentes do meio físico no qual se encontram (TUCCI, 2007).

A infiltração da água para as camadas mais internas do solo tem interferência direta na recarga dos lençóis freáticos. A depender da classificação do solo e de seus componentes, essa condução pode ser mais fácil ou dificultosa. Para solos com maiores teores de argila ou com grande compactação, torna-se bastante demorada esta recarga (SOUZA, RODRIGUES, 2014). Com a urbanização e consequente impermeabilização do solo é um problema atual e recorrente para os aquíferos e fontes de água subterrâneas, principalmente nos grandes centros urbanos, pois o abastecimento destas é proveniente da água que percola da superfície para as camadas mais internas do solo. Este processo tem grande variabilidade de tempo para ocorrer por completo, podendo levar dias ou anos (CAVALCANTI, 2013).

Para descrever a dinâmica da água no solo são necessários ensaios em laboratório e em campo que necessitam de investimento de recursos financeiros consideráveis e bastante tempo para sua execução. Por essas razões, diversos pesquisadores optam por utilizarem modelos matemáticos ou métodos indiretos que tem embasamento em dados pré-determinados do solo e têm baixo custo de utilização e aquisição (SOUZA et al., 2008). Os resultados destes modelos matemáticos podem ser utilizados como dados de entrada em outras simulações que visam quantificar a recarga das águas subterrâneas, 
destaca-se também o tempo de realização do ensaio, que pode durar algumas frações de hora e estimar a infiltração até a recarga completa do lençol freático, já que, quando o solo atinge a saturação, seu comportamento é linear (RIGHETTO, FREITAS, 2016).

Na literatura existem diversos modelos de infiltração de água no solo, como por exemplo: Green e Ampt (1911), de Kostiakov (1932), Kostiakov-Lewis (1938), de Horton (1939), Philip (1957), Swartzendruber (1987),Soil Conservation Service (SCS, Jury e Gardner, 1991), Beerkan Estimation of Soil Transfer Parameters (BEST, Lassabatère et al., 2006) e diversos tipos de ensaios utilizados para caracterizá-los como o anel simples, anel duplo e o simulador de chuva. Silva et al., (2017) mostram que é possível obter resultados pertinentes através de modelos relativamente simples de serem utilizados. A simulação de fenômenos hidrológicos fornece subsídio para estudos de projetos (como de drenagem urbana e irrigação) e de ferramentas de gerenciamento dos recursos hídricos, como a outorga e os planos de recursos hídricos. A utilização de modelos pouco confiáveis pode resultar em múltiplos problemas gerados por falsos dados de saída, como previsões e modelagens incoerentes com o fenômeno natural ou inconsistências em análises estatísticas (PEREIRA et al., 2016).

Silva Júnior et al (2016) destacam a simplicidade e eficiência do ensaio de infiltrômetro a anel simples e anel duplo para determinar a infiltração em modelos chuva-vazão, com o objetivo de obter os principais parâmetros que influenciam diretamente no escoamento superficial de uma região. A sorvidade representa a capacidade do solo em absorver água por capilaridade na ausência de efeitos gravitacionais, e predomina nos primeiros momentos da infiltração da água no solo, por isso, é um dos parâmetros mais utilizados, em conjunto com a condutividade hidráulica, para modelar o comportamento hidrodinâmico do solo (PEREIRA e THOMAZ, 2016).

Diversos autores analisaram o desempenho de modelos de infiltração da água no solo para solos arenosos e argilosos em diferentes regiões com climas diversos (HOYOS e CAVALCANTE, 2015; IGBADUNET et al., 2016; ZOLFAGHARI et al., 2012). Para cada localidade específica, existe um método que melhor pode representá-la, sendo indicado como o mais preciso para regiões com as mesmas características e condições. Apesar dos avanços, ainda não é possível determinar qual metodologia possui melhor desempenho, logo é necessário realizar ensaios de infiltração e determinar qual modelo é mais adequado para estimar a infiltração da água no solo em diferentes períodos de tempo.

Ainda pode-se destacar que as maiores variabilidades de infiltrações em solos arenosos ocorrem nos momentos iniciais da infiltração, o que dificilmente acontece em solos argilosos. Para o primeiro caso, recomenda-se fortemente a utilização do modelo de Phillip (HOYOS e CAVALCANTE, 2015). Para Igbadunet et al., (2016) os modelos de Kostiakov, Kostiakov-Lewis, Philip e Swartzendruber performam bem em solos argilosos, em oposição ao de Horton, que superestima o volume acumulado para este tipo de localidade. Para todos os métodos citados anteriormente, exceto o BEST, Zolfaghari et al., (2012) classifica-os como aplicáveis em diversos tipos de solo quando os desempenhos estatísticos do coeficiente de determinação forem superiores a $90 \%$. Os autores ainda destacam que o modelo de Kostiakov-Lewis pode ser aplicado na grande parte dos solos, obtendo grande confiabilidade na sua reprodução e aplicação.

Vários trabalhos (MOREIRA, 2014; SILVA et al., 2017; MOURA, SILVA, 2015) buscaram analisar qualitativamente a influência do solo natural e o impacto causado por sua descaracterização no cotidiano moderno da sociedade, mas poucos visam quantificar e avaliar métodos e modelos que descrevam o comportamento do solo e gerar dados numéricos de fácil manejo que visem contribuir significativamente para o avanço controlado da sociedade. Sendo assim, objetivou-se analisar o desempenho de seis modelos de infiltração de água no solo mais utilizados em uma área suscetível à alagamentos na cidade do Recife.

\section{MATERIAIS E MÉTODOS}

O estudo foi realizado no bairro da Madalena, um dos mais importantes da capital pernambucana, pois é onde encontramse diversos pontos relevantes da cidade, como o campi Benfica da Universidade de Pernambuco, o qual abrange os campus da Escola Politécnica de Pernambuco e da Faculdade de Ciências de Administração de Pernambuco, estádio de futebol, museus, supermercados entre outros, além de servir como principal acesso para outras localizações importantes como a Universidade Federal de Pernambuco e o terminal integrado de passageiros do Recife.

Moreira (2014) destaca a vulnerabilidade do bairro da Madalena e de seus residentes e transeuntes em episódios de inundações. A autora destaca que, apesar das construções de barragens para controle de cheias, como o sistema Tapacurá, a utilização dessas com o objetivo de abastecimento aliado aos maus uso e ocupação do solo intensifica esses eventos de alagamentos causados por chuvas intensas, como as de 2011 e 2013.

Oliveira e Soares (2017) classificaram o solo da região como arenoso, havendo variações de subclassificação entre areia, areia franca e franco-arenosa. Além disso, os autores avaliaram o solo em relação ao potencial de infiltração do mesmo, sendo classificado com alto potencial de infiltração, devido a ensaios de campo realizados na localidade.

A região estudada foi demarcada de acordo com a Figura 1, com linha externa de coloração preta. A área tem aproximadamente $228.000 \mathrm{~m}^{2}$, sendo cerca de $17 \%$ deste de solo natural. As subregiões delimitadas em vermelho, identificadas de 1 a 9 são os principais pontos de ocorrência de terreno natural permeável, onde foram os pontos alvos de execução dos ensaios e recolhimento das amostras. Nos pontos 1, 4, 8 e 9 a maior ocorrência de cobertura vegetal é de pequeno porte, como as gramíneas e arbustivas. Já nos pontos restantes, além da vegetação citada, também se encontram arvores de grande porte, como coqueiros e mangueiras, ou seja, típicas de regiões equatoriais umidas. 
Figura 1 - Local de estudo, indicando os principais pontos de ocorrência de solo natural, numerados de 1 a 9

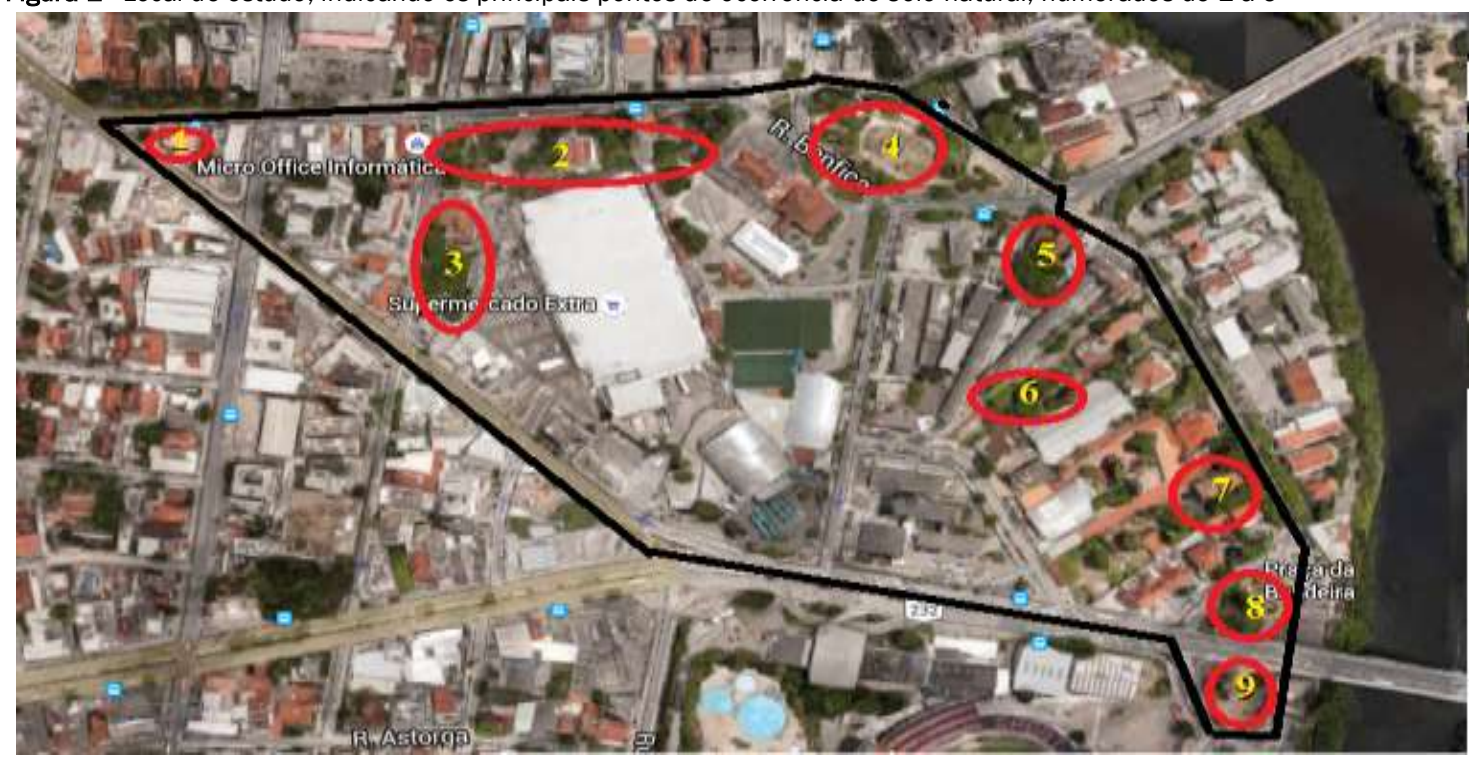

Nessas localidades (Figura 1) foram realizados os ensaios de infiltração, utilizando o infiltrômetro de anel simples, a fim de se comparar com os resultados dos seis modelos de infiltração. Para a realização desses ensaios, colocou-se um volume constante de água (300ml) no interior do anel, cronometrandose o tempo em que toda água levou para infiltrar no solo. 0 processo foi repetido até que os intervalos de tempo de infiltração de água e outra se tornasse constante, definindo assim a saturação do solo presente no interior do infiltrômetro.

Por terem um solo de mesma classificação, o tempo de duração de cada ensaio, das nove regiões, foi de aproximadamente 15 minutos. Com o ensaio de infiltração obtém-se pares ordenados de infiltração de água acumulada e tempo total decorrido do ensaio, sendo possivel construir a curva de infiltração acumulada, que é necessária para aplicação dos modelos matemáticos e a partir desses dados, estimam-se os parâmetros de cada um dos métodos com auxílio de software computacional. Neste caso foi utilizado o excel, com auxílio da ferramenta solver, que, a partir a minimização das diferenças entre os dados simulados e coletados em campo, otimizando os parâmetros necessários para aplicação de cada modelo matemático utilizado.

\subsection{Modelos de infiltração de água no solo}

\subsubsection{Método de Kostiakov (KO)}

O modelo de Kostiakov (1932) está entre um dos mais utilizados de maneira empírica, podendo ser visualizado na Equação 1.

$$
I=K_{s} t^{\vartheta}
$$

Em que $t$ é o tempo em segundos, $\vartheta$ é definida como a taxa decrescente da infiltração no decorrer do tempo, $K_{s}$ a condutividade hidráulica saturada do solo, medida em $\mathrm{mm} / \mathrm{s}$, e $I$ a infiltração acumulada, em mm.
O modelo de Horton (1939) é apresentado na Equação 2.

$$
x(t)=x_{b}+\left(x_{i}-x_{b}\right) e^{-K_{s} t}
$$

Onde $x(t)$ é a taxa de infiltração no decorrer do tempo, $x_{b}$ é a taxa mínima de infiltração, ou taxa de infiltração básica e $x_{i}$ é a taxa de infiltração inicial, medidas em $\mathrm{cm} / \mathrm{s}$.

\subsubsection{Método de Philip (PH)}

Philip (1957) desenvolveu numericamente a equação de Klute (1952), resultando na Equação 3.

$$
I=S t^{\frac{1}{2}}+K_{S} t
$$

Onde $S$ corresponde à sorvidade, medida em $m m . s^{-1 / 2}$.

\subsubsection{Método de Kostiakov-Lewis (KL)}

O modelo de Kostiakov-Lewis (LEWIS e MILNE, 1938) foi desenvolvido com o intuito de modelar tempos que tendem ao infinito. Neste caso, a infiltração tende para a velocidade de infiltração básica, ao invés de anular este parâmetro (SANTOS et al., 2016). Ela pode ser definida de acordo com a Equação 4.

$$
I=K_{s} t^{\vartheta}+x_{b} t
$$

\subsubsection{Método Soil Conservation Service (SCS)}

Desenvolvido com a finalidade de ser aplicado em simulações de longos períodos de tempo, tendo em vista que o modelo de Kostiakov não se aplicava nesta situação. Após experimentos foi possível concluir que o coeficiente independente 0.6985 pode ser acrescido a este modelo, funcionando corretamente em todas as situações (JURY, GARDNER, 1991):

$$
I=a t^{b}+0,6985
$$


Sendo $a$ e $b$ são constantes ajustadas ao modelo a partir dos dados experimentais obtidos em campo (ZOLFAGHARI et al., 2012).

\subsubsection{Método de Swartzendruber (SW)}

Swartzendruber (1987) apresentou seu modelo de acordo com a Equação 6:

$$
I=x_{c} t+\frac{c}{d}\left[1-\exp \left(d t^{0,5}\right)\right]
$$

Onde $x_{c}$ é a taxa de infiltração final, em $\mathrm{mm} / \mathrm{s}, c$ e $d$ são constantes empíricas.

\subsection{Parâmetros Estatísticos}

Para verificar a concordância entre os valores calculados e os observados, foram avaliados diferentes critérios estatísticos:

\subsubsection{Coeficiente de determinação}

O coeficiente de determinação, também chamado de $\mathrm{R}^{2}$, é uma medida de ajustamento de um modelo estatístico linear generalizado, como a Regressão linear, em relação aos valores observados. A Equação é definida em 7 (ANTONINO et al., 2004).

$$
R^{2}=\frac{\left(\sum_{i=1}^{n}\left(x_{i}-\bar{x}\right) Y_{i}\right)^{2}}{\sum_{i=1}^{n}\left(x_{i}-\bar{x}\right)^{2} \sum_{i=1}^{n}\left(Y_{i}-\bar{Y}\right)^{2}}
$$

Sendo $T_{i}$ os valores calculados pelo modelo, $M_{i}, x_{i}, Y_{i}$ os valores experimentais e $\bar{M}, \bar{x}, \bar{Y}$ a média dos valores experimentais, e N o número de determinações.

\subsubsection{Erro Quadrático Médio}

O Erro Quadrático Médio (EQM), definido na Equação 8, indica o grau de desvio entre as determinações experimentais e os valores calculados pelo modelo teórico correspondente. É expresso em porcentagem, e tende para zero quando os valores estimados e teóricos tendem a ser iguais. Este teste fornece informações de fácil entendimento no desempenho dos modelos, além de permitir uma comparação termo a termo do desvio real entre o valor calculado e o valor medido (ANTONINO et al., 2004).

$$
E Q M=\left[\frac{\sum_{i=0}^{N}\left(T_{i}-M_{i}\right)^{2}}{N}\right] * \frac{100}{\bar{M}}
$$

\subsubsection{Razão dos Desvios}

A razão dos desvios (RD), Equação 9, descreve a razão entre o espalhamento das determinações experimentais e o espalhamento dos valores calculados pelo modelo teórico correspondente, tendendo para 1 (um) quando os valores estimados do modelo teórico são consistentes (ANTONINO et al., 2004).

$$
R D=\frac{\sum_{i=0}^{N}\left(M_{i}-\bar{M}\right)^{2}}{\sum_{i=0}^{N}\left(T_{i}-\bar{M}\right)^{2}}
$$

\subsubsection{Eficiência da Modelagem}

A eficiência da modelagem (EM), definida na Equação 10, indica se o modelo teórico fornece uma estimativa melhor das determinações experimentais que o valor médio dessas determinações, tendendo para 1 (um) em simulações mais próximas da realidade (ANTONINO et al., 2004).

$$
E M=\frac{\sum_{i=0}^{N}\left(M_{i}-\bar{M}\right)^{2}-\sum_{i=0}^{N}\left(T_{i}-M_{i}\right)^{2}}{\sum_{i=0}^{N}\left(M_{i}-\bar{M}\right)^{2}}
$$

\section{Resultados e discussão}

Os valores da condutividade hidráulica do solo, em $10^{-1} \mathrm{~mm} / \mathrm{s}$, para os métodos KO, HO, PH e KL estão apresentados na Tabela 1. Vale salientar que os outros dois métodos, SCS e SW não utilizam este parâmetro nos seus modelos. Já a sorvidade, medida em $m m \cdot s^{-1 / 2}$.

Não houve variação de ordem de grandeza entre os resultados encontrados para a condutividade, além de, nos 4 métodos que utilizaram esta parametrização, corroborarem entre a localidade 8 obter os menores valores de $K_{s}$, assim como na localidade 9 convergirem para os maiores valores numéricos. Essa correlação também acontece para os valores $a$ do método SCS e para os valores $d$ do método SW.

De forma geral, todos os modelos apresentados mostraram resultados excepcionais para todas as amostras estudadas, com $\mathrm{R}^{2}$ médios superiores a 0,97, e eficiências de modelagens superiores a 0,96, conforme expostos na Figura 3. Apesar do modelo SCS ter sido desenvolvido, a princípio, para longos tempos de simulação, ele obteve desempenho satisfatório na sua simulação, de acordo com os resultados apresentados na Figura 3, apresentando uma eficiência de modelagem de 0,992. 
Tabela 1 - Valores dos parâmetros (Par.) para os métodos (Mét.) KO, HO, PH, KL, SCS e SW para cada localidade de estudo

\begin{tabular}{|c|c|c|c|c|c|c|c|c|c|c|}
\hline \multirow{2}{*}{ Mét. } & \multirow{2}{*}{ Par. } & \multicolumn{9}{|c|}{ Local } \\
\hline & & 1 & 2 & 3 & 4 & 5 & 6 & 7 & 8 & 9 \\
\hline \multirow{2}{*}{ KO } & $K_{s}$ & 1,16 & 2,42 & 7,99 & 3,34 & 3,30 & 8,10 & 5,65 & 0,78 & 15,47 \\
\hline & $\vartheta$ & 0,89 & 0,73 & 0,80 & 0,66 & 0,75 & 0,56 & 0,60 & 0,07 & 0,43 \\
\hline \multirow{3}{*}{$\mathrm{HO}$} & $x_{b}$ & 97,85 & 78,25 & 92,48 & 58,71 & 82,69 & 56,91 & 56,91 & 98,61 & 44,48 \\
\hline & $x_{i}$ & 0,00 & 0,00 & 0,00 & 0,00 & 0,00 & 0,00 & 0,00 & 0,00 & 0,01 \\
\hline & $K_{s}$ & 0,006 & 0,007 & 0,010 & 0,008 & 0,011 & 0,013 & 0,013 & 0,004 & 0,015 \\
\hline \multirow{2}{*}{$\mathrm{PH}$} & $S$ & 0,27 & 0,54 & 1,16 & 0,63 & 0,69 & 1,01 & 0,75 & 0,21 & 0,96 \\
\hline & $K_{s}$ & 0,45 & 0,21 & 0,23 & 0,12 & 0,40 & 0,10 & 0,12 & 0,08 & 0,96 \\
\hline \multirow{3}{*}{$\mathrm{KL}$} & $x_{b}$ & 0,05 & 0,03 & 0,04 & 0,02 & 0,05 & 0,02 & 0,02 & 0,03 & 0,01 \\
\hline & $\vartheta$ & 0,12 & 0,16 & 0,32 & 025 & 0,21 & 0,24 & 0,19 & 0,14 & 0,31 \\
\hline & $K_{s}$ & 27,71 & 27,04 & 22,35 & 21,87 & 23,29 & 27,43 & 24,82 & 14,67 & 32,23 \\
\hline \multirow{2}{*}{ SCS } & $a$ & 0,10 & 0,17 & 0,67 & 0,25 & 0,28 & 0,63 & 0,11 & 0,06 & 1,36 \\
\hline & $b$ & 0,91 & 0,78 & 0,64 & 0,70 & 0,77 & 0,60 & 0,83 & 0,91 & 0,45 \\
\hline \multirow{3}{*}{ SW } & $x_{c}$ & 0,05 & 0,04 & 0,07 & 0,03 & 0,07 & 0,04 & 0,03 & 0,03 & 0,02 \\
\hline & $c$ & 1,60 & 1,48 & 1,64 & 2,39 & 2,54 & 3,19 & 1,38 & 1,20 & 3,34 \\
\hline & $d$ & 0,84 & 0,49 & 0,31 & 0,52 & 0,25 & 0,53 & 0,65 & 0,03 & 0,03 \\
\hline
\end{tabular}

O método de Swartzendruber foi desenvolvido para tempos curtos, médios e longos. Apesar dos resultados obtidos pelos parâmetros estatísticos neste experimento, foi identificado que, para longas simulações, este modelo subestima a infiltração, devendo ser avaliado em diferentes tipos de solo para sua utilização correta. Por outro lado, o modelo de Kostiakov-Lewis se destacou dos demais, pois obteve correspondência muito próxima da unidade (100\% de representatividade) para 7 dos 9 locais analisados. Este modelo, por utilizar-se de um parâmetro logarítmico, descreve com bastante proximidade o comportamento da curva de infiltração da água no solo.

O modelo que obteve melhor significância na modelagem do experimento foi o de $\mathrm{KO}$, especificamente na realizada no local 4. Conforme figura 2a, a curva de infiltração simulada foi sobreposta aos pontos representativos aos ensaios de campo, e juntamente com o gráfico de dispersão, apresentam uma correlação próxima a função bissetriz, que corresponde à modelagem sem erros. Esta correlação pode ser reafirmada pelo coeficiente de determinação explicitado neste mesmo gráfico, indicado pelo $\mathrm{R}^{2}$.
Neste caso, nos momentos iniciais do ensaio, o modelo Horton não obteve adequação semelhante aos demais métodos, havendo subestimado o volume acumulado nos primeiros $10 \mathrm{~min}$ de experimento. Para simulações que exijam grande durações, como em projetos de drenagem urbana ou balanços hídricos realizados nesta região da Madalena, essa alteração pouco interfere nos resultados finais. Já em ocasiões em que sejam necessários estudos em curta duração de tempo, como os de contaminação de lençol freático, é necessário avaliar a utilização do método de Horton.

Na Figura 3 estão apresentados os diagramas de caixa dos parâmetros estatísticos utilizados na análise dos seis modelos investigados. As menores variabilidades encontradas para o coeficiente de determinação (Figura 3a) são encontradas no modelos KL, KO e SCS. Os valores médios nesses casos também são os mais próximos dos de referência. 
Figura 2 - Gráfico de modelagem do modelo com melhor desempenho (Kostiakov-Lewis - KO) e pior desempenho (Horton - HO) nas localidades 4 e 6 , respectivamente

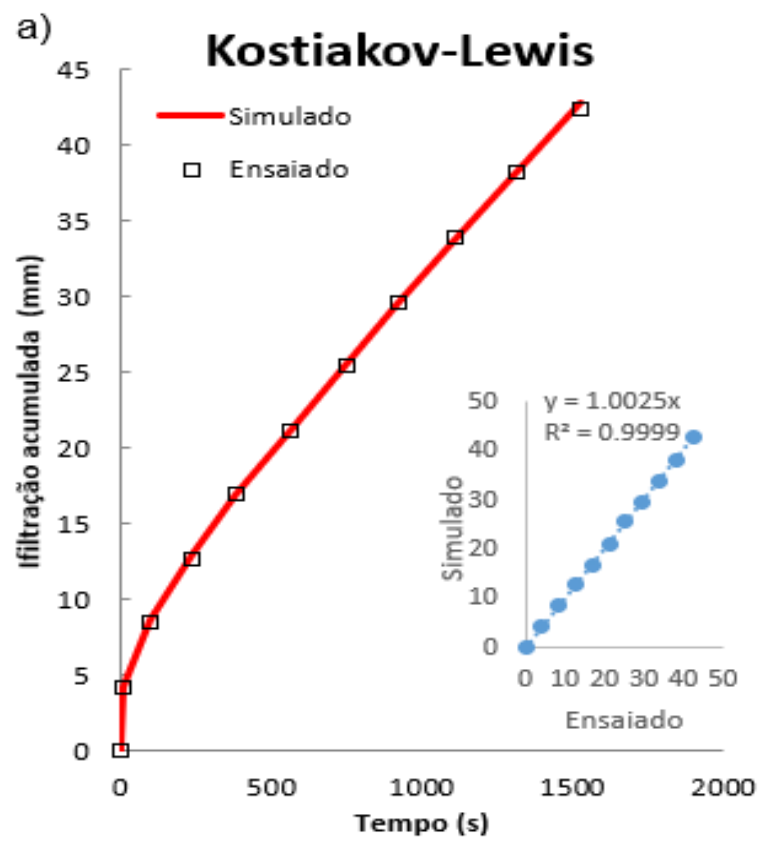

Na Figura 3b é possível identificar os valores do EQM para os seis modelos, nas 9 localidades. 0 modelo $\mathrm{KL}$ obteve o melhor desempenho, apresentando valores inferiores a $5 \%$. 0 segundo, terceiro e quarto colocados foram os modelos $\mathrm{PH}, \mathrm{KO}$ e $\mathrm{KL}$, respectivamente. Novamente o modelo $\mathrm{HO}$ obteve o menor desempenho. Já na Figura 3c pode-se visualizar que quatro dos seis modelos obtiveram todos os seus valores acima dos 0,8 com ênfase no modelo KL. Os modelos com maiores desvios foram o SW e HO, respectivamente. Este mesmo comportamento foi encontrado para a EM.

Ao analisarmos individualmente, vemos que o modelo $\mathrm{KL}$ obteve a menor variabilidade e maior proximidade entre os valores dos pontos médios e os valores dos pontos de referência, logo, obteve o melhor desempenho estatístico, comparado aos demais.

Já o modelo de HO obteve maior variabilidade nos 4 indicadores, mostrando desempenho inferior aos demais. No coeficiente de determinação, na Razão dos Desvios e na Eficiência de Modelagem, suas medianas foram bem próximas ao terceiro quartil, indicando um comportamento tendencioso por parte dos dados. Ainda assim a variação dos dados é considerada baixa. Neste método, as discrepâncias máximas para a variabilidade foram de 0,03 para $\mathrm{R}^{2}, 3,80$ para o $\mathrm{EQM}, 0,08$ para $\mathrm{RD}$ e 0,03 para EM.

Os menores índices de desempenho alcançados foram os relativos aos erros quadráticos médios, que indicam os erros associados a previsão do sistema. Para os modelos de KL, KO, SCS e $\mathrm{PH}$ essas diferenças não chegam a 2 pontos, considerados

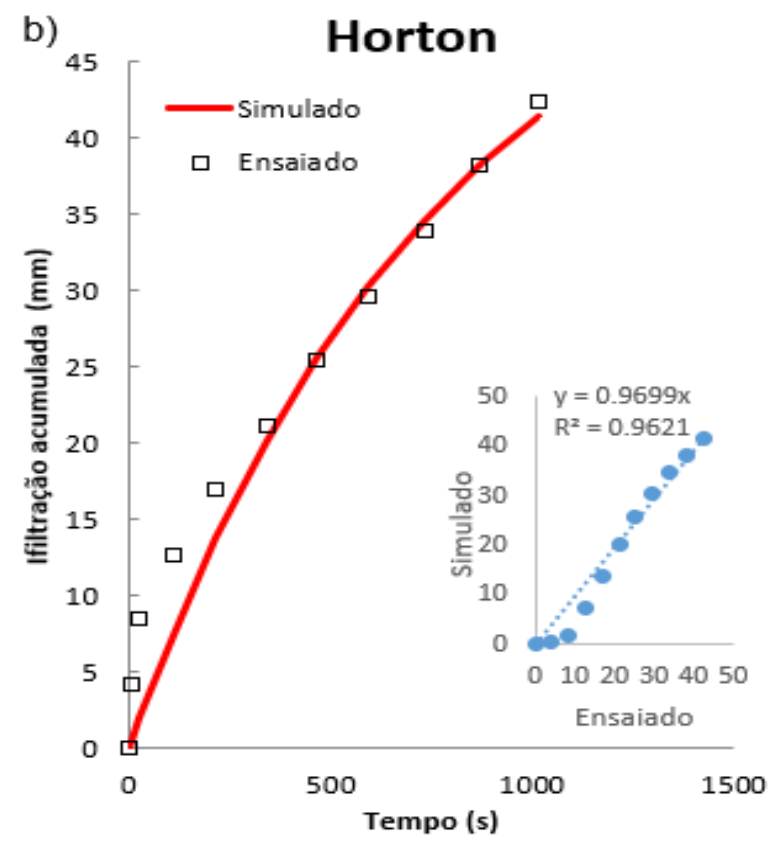

erros muito baixos (SILVA et al., 2016).

De acordo com a Figura 3, é possível visualizar que os valores médios do $\mathrm{R}^{2}$ para todos os modelos analisados foram superiores a 0,9. Os modelos que apresentaram maior e menor $\mathrm{R}^{2}$ médios foram o $\mathrm{KL}\left(\mathrm{R}^{2}=0,998\right)$ e o $\mathrm{HO}\left(\mathrm{R}^{2}=0,975\right)$, respectivamente. Estes resultados foram semelhantes aos encontrados por Zolfaghariet al., (2012), que analisaram o desempenho de modelos de infiltração para 95 diferentes localidades. Eles obtiveram valores de $\mathrm{R}^{2}$ entre 0,992 e 0,998, sendo o melhor desempenho encontrado para o modelo $\mathrm{KL}$ e o modelo de menor $\mathrm{R}^{2}$ para o modelo $\mathrm{HO}$.

Mishra et al., (2003) utilizou os métodos empíricos para modelar a infiltração da água no solo em 23 localidades diferentes. Os resultados indicaram que o modelo de Philip não é apropriado para simulações em grandes intervalos de tempos, para as localidades estudadas. Na região da Madalena, para um solo do tipo predominantemente arenoso, este modelo possuiu excelente correspondência com os testes realizados em campo.

Os valores médios da RD ficaram próximos de um, indicando que o espalhamento entre os valores médios e calculados possuem comportamentos semelhantes. A ordem classificação do primeiro ao sexto, quanto aos valores da RD, foram: $\mathrm{KL}(\mathrm{RD}=$ $1,000), \mathrm{KO}$ e SCS (ambos com RD = 0,964), $\mathrm{PH}(\mathrm{RD}=0,951)$, $\mathrm{HO}(\mathrm{RD}=0,842)$ e SW $(0,821)$. Os valores médios determinados pelos modelos para a EM ficaram entre 0,962 e 1,000. Novamente, os melhores valores foram encontrados para o modelo $\mathrm{KL}$ e o modelo com EM foi o $\mathrm{HO}$. 
Figura 3 - Boxplot dos indicadores estatísticos - a) R²; b) EQM; c) RD; d) EM
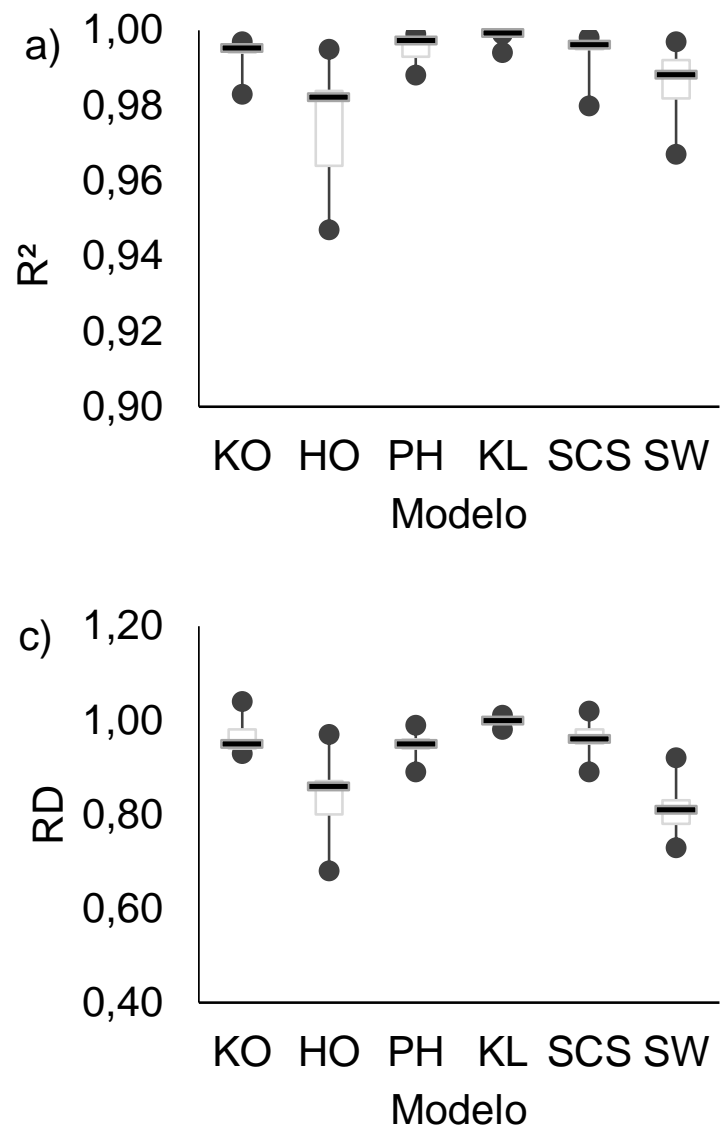

Diferentes graus de desvios foram determinados pelos modeIos analisados. O modelo que apresentou, em média, o menor desvio, e consequentemente o menor médio de EQM foi o $\mathrm{KL}$ (EQM $=1,84 \%)$. O segundo menor valor coube ao modelo SCS $(\mathrm{EQM}=4,40 \%)$. Em terceiro lugar ficou o modelo SCS (EQM = $4,78 \%)$. Com valores de EQM de $5,12 \%$ e 9,39\%, os modelos KO e SW e ficaram na quarta e quinta colocação, respectivamente. 0 maior valor encontrado para o EQM foi determinado pelos dados do modelo HO, com um EQM de 11,47\%.

Em geral, parte do desempenho dos modelos pode ter sido afetada pelo tempo de infiltração, uma vez que o solo analisado possui textura arenosa. Segundo Sihag et al. (2017), os erros podem ser minimizados a partir do incremento temporal do experimento, uma vez que os modelos KO, KL e SCS têm melhor desempenho para simulações com tempos mais longos.

\section{CONSIDERAÇÕES FINAIS}

Os modelos de infiltração de água no solo possuem grande relevância em diversos estudos de modelagem referentes ao comportamento hidrodinâmico do solo. Como existem diversos métodos aplicáveis à simulação da infiltração da água do solo, e considera-se algum ou alguns modelos mais adequados para cada região específica, o presente estudo destaca seis modelos que podem ser utilizados em uma região central e relevante da cidade do Recife. Neste caso, para uma região suscetível a alagamentos, que contém solos caracteristicamente areno-
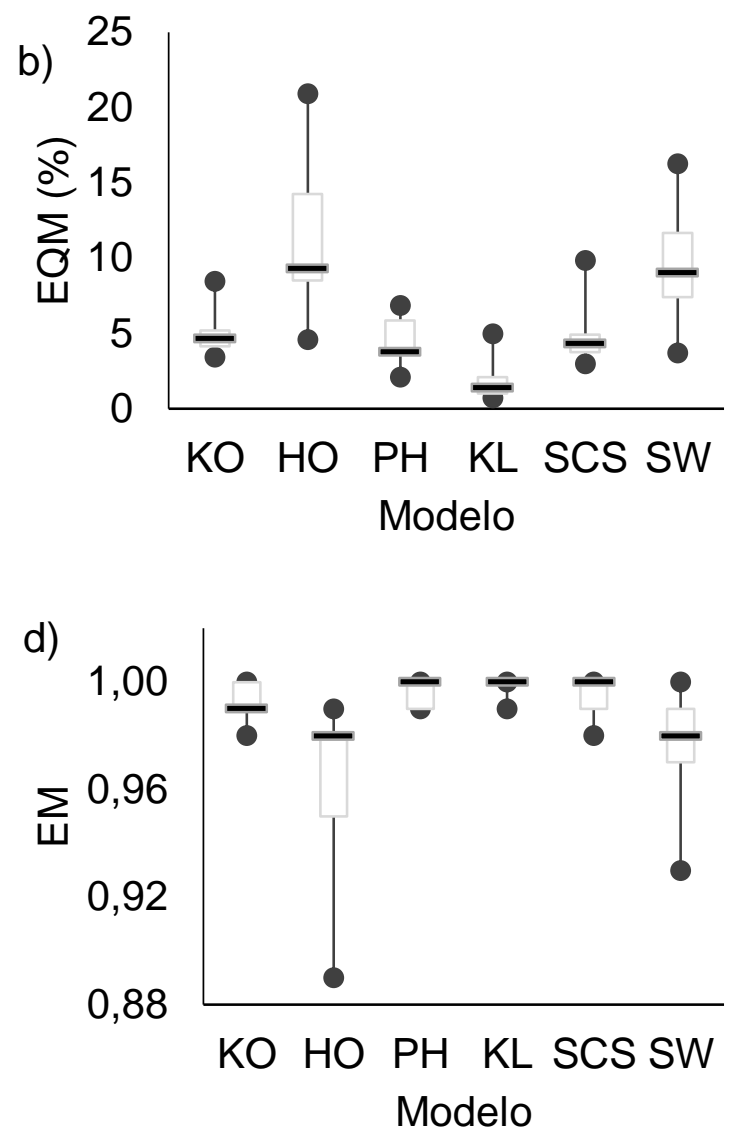

sos, foram avaliados seis métodos usuais e de simples aplicação.

A análise realizada através dos parâmetros estatísticos $\left(R^{2}\right.$, EQM, RD e EM) mostrou que todos modelos podem ser utilizados para representar a área estudada.

Mesmo que o tempo do ensaio de infiltração realizado tenha sido curto, 15 minutos, os modelos KO, KL e SCS que costumam apresentar um melhor desempenho para simulações com tempos mais longos se mostraram muito eficazes para os solos das nove regiões estudadas.

Os resultados de desempenho máximo e mínimo, para os modelos estudados, em solos arenosos, corroborou com os encontrados por Hoyos e Cavalcante, (2015) e Igbadunet et al., (2016). Para aplicação do PH, KL, SW, SCS, os resultados também condizem com Zolfaghari et al., 2012, já o modelo HO, que resultou em um acréscimo no volume de infiltração acumulada, subestimou este volume nesta localidade. Fatores como a compactação do solo influenciam diretamente neste resultado.

Dentre os métodos, o que mais se destacou foi o $\mathrm{KL}$, com $\mathrm{R}^{2}$ de $99,8 \%$ e um EQM de 1,84\%. O modelo de Horton foi o pior deles, pois apresentou um coeficiente de determinação de 97,5\% e um Erro quadrático médio de 11,471\%, mas ainda assim, pode ser utilizado para avaliar os solos das regiões estudadas. 


\section{AGRADECIMENTOS}

0 primeiro autor deste trabalho agradece à FACEPE (Fundação de Amparo à Ciência e Tecnologia do Estado de Pernambuco) pelo financiamento da bolsa (BIC-0669-1).

\section{REFERÊNCIAS}

ANTONINO, A. C. D.; SOARES, W. A.; SILVA, E. B.; LIMA, J. R. S.; NETTO, A. M.; LIMA, C. A. B. O. Utilização do método inverso para a caracterização hidrodinâmica de um neossoloflúvico. Revista Brasileira de Recursos Hidricos, v. 9, n.3, p. 81-87, 2004.

CAVALCANTI, H. F. Consequências da urbanização desordenada em área de nascente. Revista Didática Sistêmica, v.15, n.1, 2013.

GREEN, W. H.; AMPT, G. A. Studies on soil physics-1. The flow of air and water through soils. Journal of Agricultural Science, v. 4, n. 1, p. 1-24, 1911.

HOYOS, E. M.; CAVALCANTE, A. L. B. Sensitivity Analysis of One-Dimensional Infiltration Models. EJGE, v. 20, n. 10, p. 4313-4324, 2015.

HORTON, R.E. Analysis of runoff-plot experiments with varying infiltration capacity. Transactions American Geophysical Union, v.20, p. 693 711, 1939.

IGBADUN, H. E.; OTHMAN, M. K.; AJAYI, A. S. Performance of Selected Water Infiltration Models in Sandy Clay Loam Soil in Samaru Zaria. Global Journal of Researches in Engineering, v. 16, n.4, 2016.

JURY, W.A.; GARDNER, W. R. Soil physics. 5th ed. New York (NY), John Wiley \& Sons, 1991.

KLUTE, A. A numerical method for solving the flow equation for water in unsatured materials. Soil Sci. v.73, p. 105-116, 1952.

KOSTIAKOV, A. N. On the dynamics of the coeficiente of water percolation in soils and the necessity of studying it from dynamic point of view for purposes of amelioration. 6th Committee of International Society of Soil Science. Paris: International Soil Science Society, v. 6, p. 17 21, 1932.

LASSABARÈRE, L.; ÂNGULO-JARAMILLO, R.; UGALDE, J.M.S.; CUENCA, R.; BRAUD, I.; HAVERKAMP, R. Beerkan Estimation of Soil Transfer Parameters through Infiltration Experiments - BEST. Soil Sci., v.70, p. 521-532, 2006.

LEWIS, M. R.; MILNE, W. E. Analysis of border irrigation. Agriculture Engineering, v. 19, n. 6, p. 267-272, 1938.

MISHRA, S. K.; TYAGI, J. V.; SINGH, V. P. Comparison of infiltration models. Hydrological processes, v. 17, p. 2629-2652, 2003.

MOREIRA, M. F. “Tapacurá estourou!”: a vulnerabilidade da cidade anfíbia (Recife-PE) aos episódios de inundações e o bairro da Madalena. Tese (doutorado) - Universidade Federal de Santa Catarina, Centro de Filosofia e Ciências Humanas, Doutorado em Ciências Humanas, Florianópolis, 2014.
OLIVEIRA, D. B. C.; SOARES, W. A. Desempenho de modelos de infiltração tridimensional de água no solo. Revista Diálogos, v. 2, n. 18, p. 519-544, 2017.

PEREIRA, D. R.; ULIANA, E. M.; MATINEZ, M. A.; SILVA, D. D. Desempenho de um modelo hidrológico concentrado e de um semidistribuído na predição de vazões diárias. Irriga - Brazilian Journal of irrigation and drainage. v. 21, n. 2, p. 409-424, 2016.

PEREIRA, A.A.; THOMAZ, E.L. Condutividade hidráulica e sorvidade em áreas sob diferentes sistemas de uso e manejo. Simpósio Nacional de Geomorfologia, 11, 2016, Maringá.

PHILIP, J.R.; The Theory of Infiltration: 1. The Infiltration Equation and its solution. Soil Sci, v.83, p.345-357, 1957.

RIGHETTO, A. M.; FREITAS, B. Hydrological modeling of a detention reservoir: flood control and aquifer recharge. Revista Brasileira de Recursos Hídricos, v.21, n.4, p.752-765, 2016.

SANTOS, T.E.M.; SOUZA, E.R.; MONTENEGRO, A.A.A. Modeling of soil water infiltration with rainfall simulator in different agricultural systems. Revista Brasileira de Engenharia Agrícola e Ambiental, v.20, n.6, p. 513-518, 2016

SIHAG, P.; TIWARI, N.K.; RANJAN, S.;Estimation and inter-comparison of infiltration models. Water Science, v.31, p.34-43, 2017.

SILVA, M. R.; SANTOS, L. B. L.; SCOFIELD, G. B.; CORTIVO, F. D. Utilização de Redes Neurais Artificiais em Alertas Hidrológicos: Estudo de Caso na Bacia do Rio Claro em Caraquatatuba, Estado de São Paulo. Anuário do Instituto de Geociências - UFRJ, v.39, n.1, 2016.

SILVA, N. F.; CUNHA, F. N.; FILHO, F. R. C.; MORAIS, W. A.; CUNHA, E. S.; ROQUE, R. C.; ALVES, D. K. M.; TEIXEIRA, M. B. Métodos para estimativ da infiltração de água em um latossolo sob plantio direto e convencional. Global Science Technology, v.10, n.1, p.169-176, 2017.

SILVA JÚNIOR, M.A.B.; SILVA, S.R.; SOARES, W.A. Parâmetros de infiltrabilidade em solo urbano para modelo chuva-vazão. Fórum Ambiental da Alta Paulista, v.12, n.3, p.135-143, 2016.

SOUZA, E. S.; ANTONINO, A. C. D.; JARAMILLO, R. A.; NETTO, A. M. Caracterização hidrodinâmica de solo: Aplicação do método Beerkan. Revista Brasileira de Engenharia Agrícola e Ambiental, v.12, n.2, p.128-135, 2008.

SOUZA, F. A.; RODRIGUES, S. C. Estimativa de recarga do lençol freático em solos sobre granito e gabros alcalinos na alta bacia do rio dos Boise m Iporá - GO. Ambiciência, v.10, n.2, 2014.

SWARTZENDRUBER, D. A quasi solution of Richard's equation for the downward infiltration of water into soil. WaterResourcesResearch, v.23, p.809-817, 1987.

TUCCI, C. E. M. Hidrologia: Ciência e Aplicação, Porto Alegre, ABRH, 2007.

ZOLFAGHARI, A.A.; MIRZAEE, S.; GORJI, M. Comparison of different models for estimating cumulative infiltration. International Journal of Soil Science, v. 7, n. 3, p. 108-115, 2012. 